\title{
AUTOMATED ASSAY AND DIFFERENTIAL MODEL OF WESTERN HONEY BEE (APIS MELLIFERA) AUTOGROOMING USING DIGITAL IMAGE PROCESSING
}

Carl Giuffre", Sharon R. Lubkin",2, and David R. Tarpy ${ }^{3,4}$

'Biomathematics Graduate Program, North Carolina State University, Raleigh NC 27695-8213

${ }^{2}$ Department of Mathematics, North Carolina State University, Raleigh NC 27695-8205

${ }^{3}$ Department of Entomology and Plant Pathology and ${ }^{4}$ W.M.Keck Center for Behavioral Biology, North Carolina State University, Raleigh NC 27695-7613

Author for correspondence; EMAIL: cjgiuffr@ncsu.edu; TEL: (919) 513-1153

(C) 2016. This manuscript version is made available under the Elsevier user license http://www.elsevier.com/open-access/userlicense/1.0/ 


\section{Abstract}

In animals, self-grooming is an important component of their overall hygiene because it reduces the risk of disease and parasites. The European honey bee (Apis mellifera) exhibits hygienic behavior, which refers to the ability of the members of a colony to remove diseased or dead brood from the hive. Individual grooming behavior, however, is when a bee grooms itself to remove parasites. While both behaviors are critical for the mitigation of disease, hygienic behavior is overwhelmingly more studied because, unlike grooming behavior, it has a simple bioassay to measure its phenotype. Here, we develop a novel bioassay to expedite data collection of grooming behavior by testing different honey bee genotypes (stocks). Individual worker bees from different commercial stocks were coated in baking flour, placed in an observation arena, and digitally recorded to automatically measure grooming rates. The videos were analyzed in MATLAB, and an exponential function was fit to the pixel data to calculate individual grooming rates. While bees from the different commercial stocks were not significantly different in their grooming rates, the automation of grooming measurements may facilitate future research and stock selection for this important mechanism of social immunity.

Keywords: grooming behavior, honey bees, social insects, image processing 


\section{Graphical Abstract}

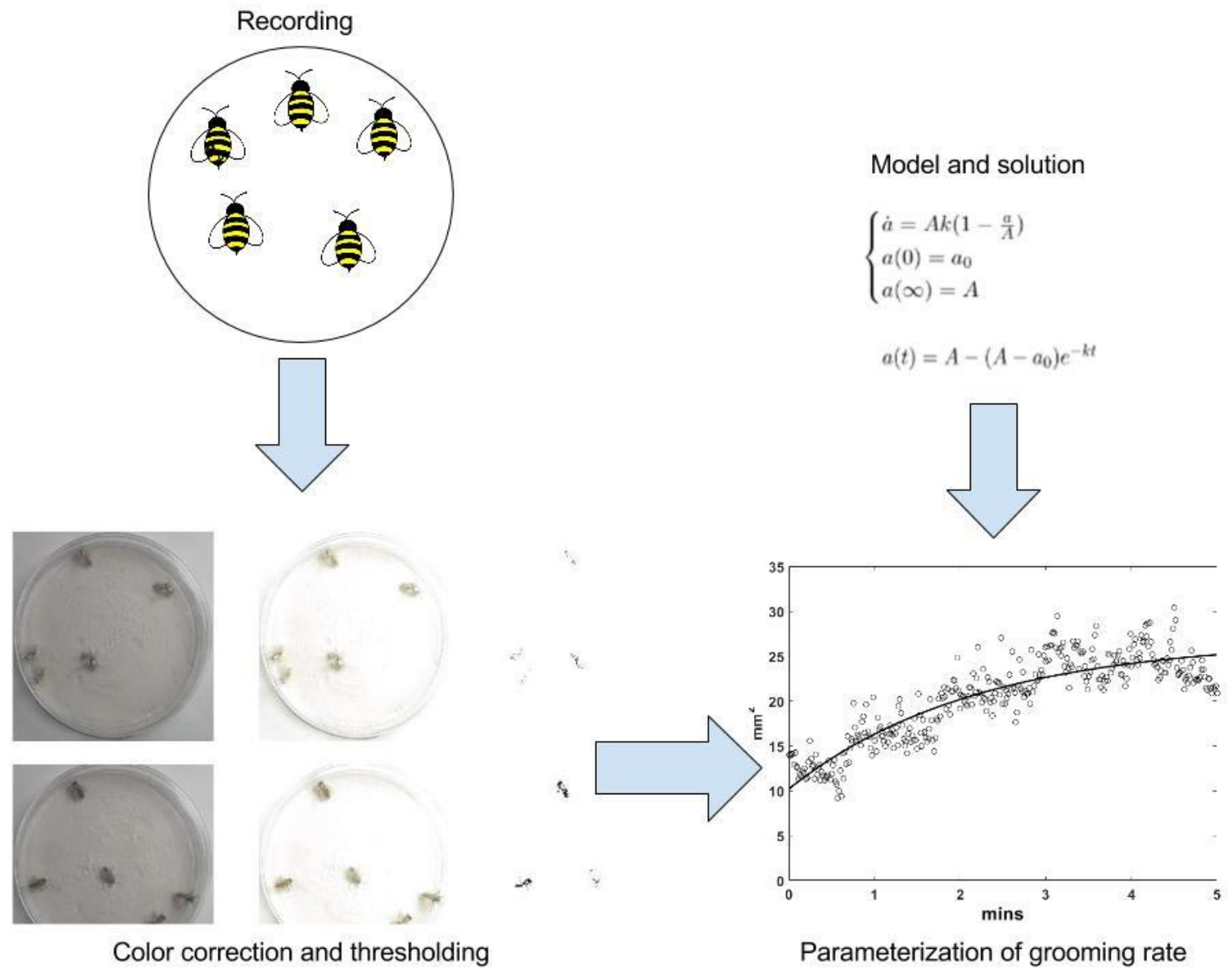




\section{Introduction}

The European honey bee (Apis mellifera) belongs to the insect order Hymenoptera, which includes many of the highly eusocial insect societies (ants, bees, and wasps). As in all organisms, honey bees have their own individual immune defenses. However, social groups increase the complexity of their relationships with parasites and pathogens (Schmid-Hempel, 1998). On the one hand, pathogens can spread more readily when high concentrations of susceptible host are in close proximity and (usually) in stable environments. On the other hand, social insects may also exhibit so-called social immunity (Cremer et al., 2007), which is the collective ability of a society to deal with parasites either behaviorally or via innate immune responses. In honey bees, allogrooming (Evans and Spivak, 2010), resin collection (Simone et al., 2009), and the removal of diseased or dead brood (Spivak and Reuter, 2001) have all been identified as behavioral mechanisms of social immunity in honey bee colonies. Techniques for improving both individual and social immunity in honey bee stocks should, therefore, have strong economic implications for commercial breeding programs.

Commercial honey bee stocks exhibit a spectrum of traits across many important characteristics, such as honey production, winter hardiness, disease resistance, hygienic behavior, and grooming behavior (Winston, 1987). Different commercial stocks of bees exist naturally and through artificial breeding (e.g., Minnesota Hygienic, Italian, Russian, Carniolan, and Varroa Sensitive Hygiene). In particular, the hygienic and grooming behaviors have been the topics of interest for many beekeepers because of what they imply for the longevity of the colony. Hygienic behavior in the strict sense refers to the colony's ability to mitigate diseases by removing diseased or dead brood from the colony, varroa mites from infested larvae, or foreign objects from the hive (Spivak and Reuter, 2001). Grooming behavior, in contrast, refers to an individual bee grooming herself to remove parasites from the exterior of her body or that of other honey bees (Winston, 1987). 
Modern assays to measure hygienic behavior have proven useful in breeding programs (Spivak and Reuter, 2001). One such assay focused on manually distinguishing classes of observable behavior of individual honey bees after being exposed to the external parasite Varroa destructor (Aumeier, 2001; Guzman-Novoa et al., 2012). Manual observation assays have an unfortunately laborious bottleneck, requiring repeated human observation that can be very time consuming. Other assays involve counting chewed mites dropped onto sticky-board placed beneath the hive (Arechavaleta-Velasco and Guzmán-Novoa, 2001; Andino and Hunt, 2011). Exposure to external parasites elicits a very confined set of behaviors, specific to parasite response, which may not be indicative of grooming as a function of pathology resistance in general. More recent studies have explored grooming as a function of varroa control (Pritchard, 2016), although very little has been done to study the generalized act of selfgrooming (also known as autogrooming) in the absence of parasitic stimulus, which may play a key role in colony-level disease pathology.

The seminal work done by Tu et. al (2016) has established both the need for and the capability of technology in automated, behavioral studies of pollinators. More broadly, the overall objective of this study was to develop a novel bioassay that may alleviate the severe bottleneck and constraints of acquiring precise data on grooming behavior. By automating and accurately quantifying grooming rates of individual bees without direct human observation (which is both time consuming and subject to inter-individual measurement error), our aim is to address this important—but largely neglected—behavioral mechanism of social immunity in honey bees.

\section{Materials and Methods}

\subsection{Experimental colonies}

Queens from commercial stocks of Italian, Russian, and Minnesota Hygienic honey bees were obtained through commercially available queen breeders. The hives were located at the 
Lake Wheeler Honey Bee Research facility located in Raleigh, NC. Queens were introduced to their respective colonies using conventional apicultural practices (Büchler et al., 2013). Since a full brood rearing cycle lasts approximately three to four weeks, the colonies were allowed 4 weeks to ensure that the emerging broods were from the appropriate genetic source.

Frames of newly emerging workers were gathered from 18 different colonies (6 frames

from each commercial stock), and stored in an incubator set at brood nest conditions $\left(34^{\circ} \mathrm{C}\right.$ and $\sim 50 \% \mathrm{RH}$ ) for approximately 12 hours. Roughly 300 'callow' honey bees from each frame were then marked on their thorax with colored paint (Testors). The marks were color-coded so that they could be identified for both the age and stock of each individual. The bees were then introduced to separate, foreign colonies ("common gardens"), thereby controlling for the effects of colony environment and social dynamics on adult behavior. Approximately 3,000 honey bees, in total, were marked in this manner. To control for seasonal factors, all cohorts were marked within a three-week window of time, spanning from late June through early July.

Bees that are one week old are typically identified as 'nurse bees' (Winston, 1987), as their behavior tends to focus on brood rearing, nursery maintenance, pathogen defense, and grooming. Thus, bees were gathered at approximately one week of age, when their grooming behavior would be most prevalent.

\subsection{Setup and Recording}

On recording day, the desired bees were collected from their respective common gardens. Groups of five bees were collected using soft forceps, and placed into a specially modified test tube with multiple holes for ventilation. The tubes were kept in an incubator, set at $34{ }^{\circ} \mathrm{C}$, until it was time for that group to be recorded. At that time, the group was taken from the incubator, and a small quantity of all-purpose wheat flour (Gold Medalтм) was added. The bees were tossed in the flour for about one minute, or until fully coated. Once the bees were fully coated in flour, they were quickly transferred to a $125 \mathrm{~mm}$ petri dish using soft forceps. To 
facilitate, the bees were temporarily dumped onto a coffee filter, where they could more easily be gathered.

Honey bee grooming was then recorded. To expedite data collection, two cameras simultaneously recorded two experiments being run at the same time. All recordings took place in a dark room with Sony Handycams mounted approximately 10 \% inches (27 centimeters) above the petri dishes containing the experimental bees. Four utility light clamps (Coleman) were installed with 10W (60W-equivalent) LED light bulbs (Lighting Science) and arranged in a square on the perimeter of both experiments. This provided uniform lighting, which reduced both the reflective glare from the tops of the petri dish and shadows generated by the bees. Beneath the petri dishes was an even layer of the same flour in which they had been coated. As this required separate recording devices, it was important to color calibrate each video sequence, and an X-Rite Color Checker was also present in the recording area, which enabled pixel color calibration of the recordings (Figure 1). 


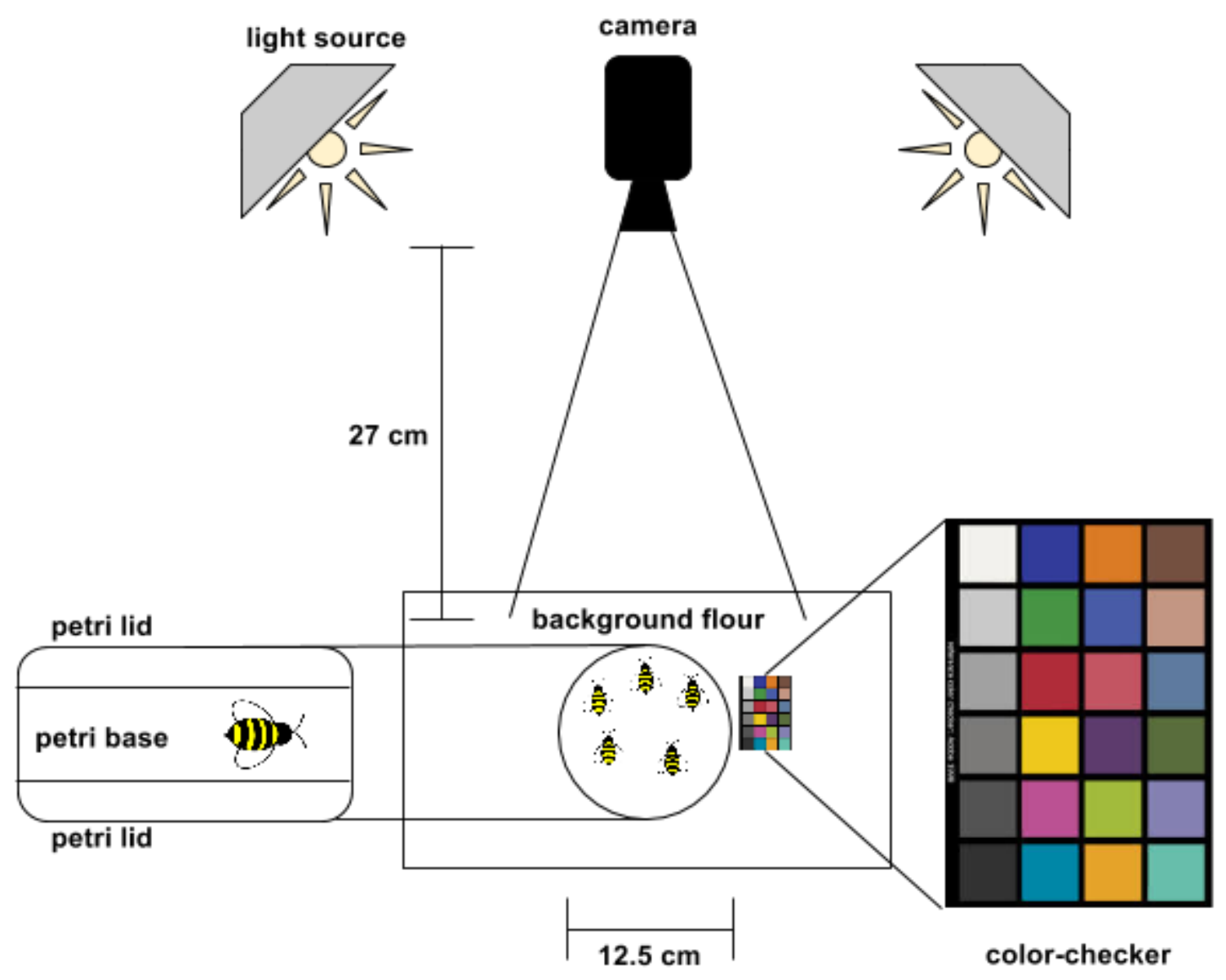

Figure 1 Experimental setup. Five honey bees were placed in a petri dish with a diameter of $12.5 \mathrm{~cm}$. Beneath this dish was an inverted petri dish lid with a small bed of flour ( $50 \mathrm{~mm}$ deep). A camera was mounted $27 \mathrm{~cm}$ directly above the petri dish, and a color-checker was placed in the recording area. Four utility lamps were mounted in a square surrounding the recording area, angled to minimize glare off the petri dish and maximize illumination of the bees

The bees were recorded for 10 minutes at 60 frames per second, with 2 minutes of buffering at the beginning of each video, for a total recording time of approximately 12 minutes. A total of 105 videos were recorded in a span of three weeks. Of these, 9 of the videos were discarded due to events such as defecation or bee death, leaving a total of 96 videos for analysis.

\subsection{Image Analysis}


Once the videos were recorded, an algorithm written in-house in MATLAB (The MathWorks) was used to analyze the video data. Our algorithm follows three steps which require minimal user intervention: (1) Frame extraction, (2) Color correction, (3) Thresholding/pixel counting and pixel-to-metric conversion (Figure 2).

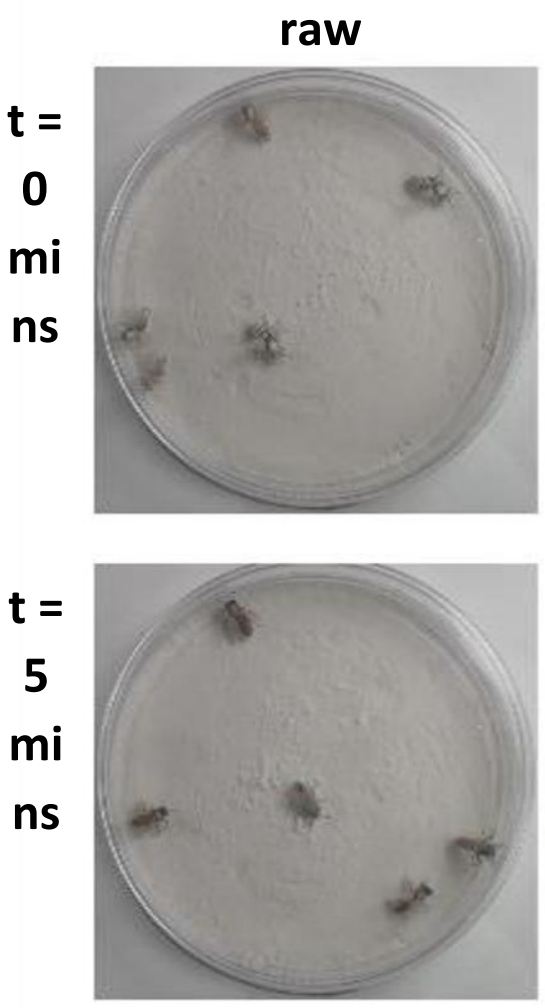

color-adjusted
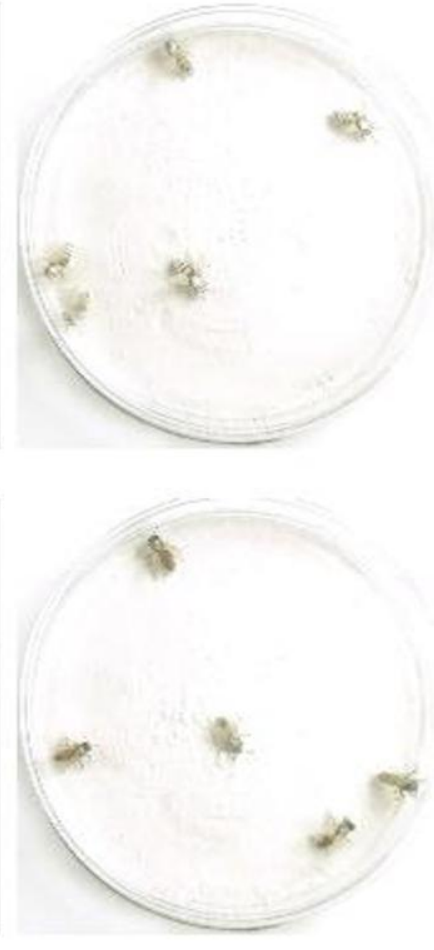

thresholded
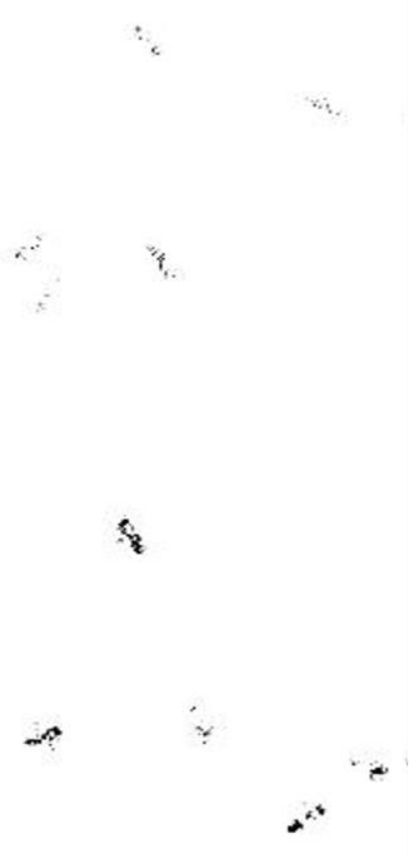

Figure 2 Steps in the processing algorithm: (left) raw images, (middle) color adjusted images, and (right) binary images. The top and bottom rows are images characteristic of the starts and ends of our experiments, respectively.

One goal of this study was to provide an algorithm that could be performed across a variety of personal computers, recording devices, and lighting arenas.

Color correction. Our setup enabled us to simultaneously record two experiments in the same twelve-minute timeframe. However, once the frames were extracted, it was important to color-correct across the multiple recording devices, to ensure accurate data analysis. Pixel 
data were compared to the expected color values of an X-Rite Color Checker using polynomial regression analysis (Hardeberg, 2001). This technique removes the device-dependence of the data, and enables data to be compared, even though separate recording devices were used. A third-degree polynomial was chosen, since reduction in standard error was significant compared to linear or quadratic fits.

Thresholding. Next, the corrected images were thresholded to obtain the binary pixel data. The images were examined using the HSV computer color spectrum and thresholded on the $\mathrm{V}$-channel. As honey bees were recorded, they moved around the petri dish and cast shadows against the flour background. Since shadow-colored pixels are dark, and the goal of this assay was to measure dark pixels as a function of grooming, it was important to estimate the threshold based on the shadows being cast by the honey bees. Thresholding on $\mathrm{V}$ at a value of 0.6275 enabled us to conservatively distinguish between background flour, shadows, and the bees. This value was obtained using ImageJ (Schneider et al., 2012) on selected frames from the start and finish of prototypical experiments.

Scaling. The thresholded pixels were counted every 100 frames. These pixel counts were then scaled to square millimeters using the known measurements of the X-Rite Color Checker as a scale. This produced a time series a(t) for each experiment.

Efficiency. Performing mathematical operations on entire video sequences is computationally time-consuming on most personal computers. Therefore, every 100 frames were extracted from each video sequence. Initially, the algorithm was performed on the full 10 minutes of video footage. However, in most cases, the measurable grooming occurred within the first 5 minutes of the video. Therefore, as another efficiency measure, only the first five minutes of each video were used in analysis.

\subsection{Mathematical Model}


The background of the recording area was the same color as the flour in which the bees were coated. Therefore, the recorded pixel data were treated as binary data where only nonflour colored pixels were counted. We were interested in formulating a model that captured the dynamics of the honey bees as they transition from flour-coated, or "dirty", to clean.

The model needed to be characteristic of the observed behaviors. One behavior observed was that the bees engaged strictly in autogrooming, despite the presence of 4 other bees. Second, honey bees would rigorously groom themselves until they were mostly clean, and this process was quite rapid. Once the bees were mostly clean, the emphasis changed from autogrooming to other behaviors. Finally, since the heaviest grooming occurs at the start of the experiment, a model that parametrized how coated the bees actually were when the video recordings started was important. These assumptions and observations can be expressed simply as

$$
\left(\begin{array}{c}
\text { rate of change of } \\
\text { flour-covered area }
\end{array}\right) \text { is proportional to }\left(\begin{array}{c}
\text { flour-covered } \\
\text { area }
\end{array}\right)
$$

Writing $A$ as the (fixed) total area of the clean honey bees $\left(\mathrm{mm}^{2}\right), a_{0}$ the initial cleanliness $\left(\mathrm{mm}^{2}\right)$, and $k$ as the grooming rate (mins ${ }^{-1}$ ) converts the "word equation" into the differential equation

$$
\dot{a}=A k(1-a / A) \text { with } a(0)=a_{0} \text { and } \lim _{t \rightarrow \infty} a(t)=A .
$$

This has solution

$$
a(t)=A-\left(A-a_{0}\right) \exp (-k t)
$$

illustrated in Fig. 3a. In the context of our grooming assay, $\lim _{t \rightarrow \infty} a(t)=A$ represents the assumption that if an experiment was theoretically allowed to run forever, then eventually $a(t)$, the area of the clean honey bees at time $t$, will asymptotically approach the quantity $A$. 
Since honey bees range from $50 \mathrm{~mm}$ to $150 \mathrm{~mm}$ in length, it was important to normalize by bee size. Defining $\varphi(t)=a(t) / A$, the fraction of bee area which is clean, and $\varphi_{0}=a_{0} / A$, the fraction clean at the start of the experiment, gives the resulting non-dimensional differential equation

$$
\dot{\varphi}=k(1-\varphi) \text { with } \varphi(0)=\varphi_{0} \text { and } \lim _{t \rightarrow \infty} \varphi(t)=1
$$

with solution $\varphi(t)=1-\left(1-\varphi_{0}\right) \exp (-k t)$, illustrated in Fig $3 \mathrm{~b}$, where $k$, again, is the grooming rate $\left(\right.$ mins $\left.^{-1}\right)$. The condition $\lim _{t \rightarrow \infty} \varphi(t)=\varphi_{0}$ is defined in a manner similar to the dimensional differential equation, using proportions of clean bee area, rather than total bee area.

\section{Results}

\subsection{Time series}

The time series $\mathrm{a}(\mathrm{t})$ of the data were then fitted to the dimensional model to obtain the parameters $a_{0}, A$, and $k$ for each experiment. The global data were highly stochastic due to bee motion and other behaviors, so parameter fitting was done with the MATLAB package Isqnonlin (The MathWorks). Figure 3c shows a typical time series and resulting curve from nonlinear 
least-squares fit. These data were then converted to their non-dimensional counterparts, using
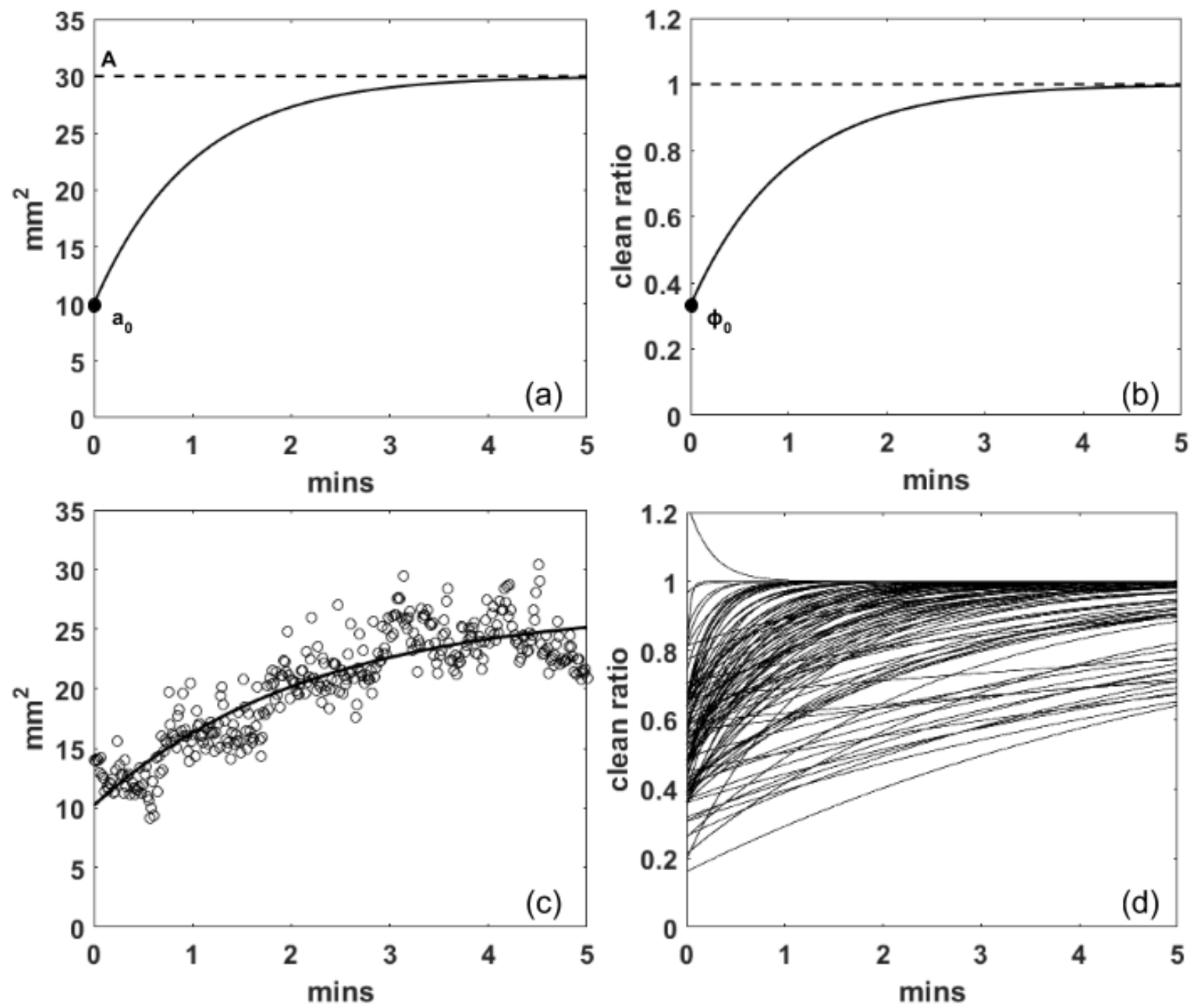

Figure 3 Data and curve fitting of bee cleanliness over time. (a) Dimensional and (b) non-dimensional areas can be approximated by the expressions $\boldsymbol{a}(\boldsymbol{t})=\boldsymbol{A}-\left(\boldsymbol{A}-\boldsymbol{a}_{\mathbf{0}}\right) \exp (-\boldsymbol{k} t)$ and $\varphi(t)=1-$ $\left(1-\varphi_{0}\right) \exp (-k t)$ respectively, where the parameter $\boldsymbol{A}$ represents the long-term asymptotically clean area of five honey bees $\left(\mathrm{mm}^{2}\right), \boldsymbol{a}_{0}$ represents the area of five honey bees that were clean at the start of the experiment $\left(\mathrm{mm}^{2}\right), \boldsymbol{\varphi}_{0}$ represents the ratio of five honey bee areas that were clean at the start of the experiment, and $\boldsymbol{k}$ is the grooming rate $\left(\right.$ time $\left.^{-1}\right)$. Parameters and curves in (a) and (b) are for illustration only. (c) Individual time series are highly stochastic due to bee motion and grooming behaviors, but the fit to model curves from (a) and (b) yields the individual grooming rate parameter $\boldsymbol{k}$, quantifying the

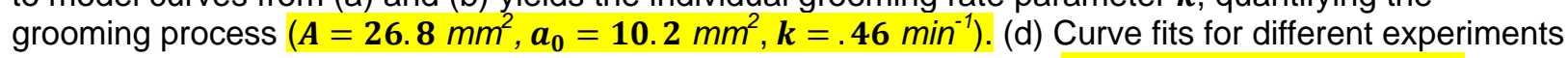
may have very different grooming rates and starting cleanliness ratios. One curve appears to have decreasing cleanliness, but is treated as an outlier (see Discussion).

the parameters from the dimensional fit. 


\subsection{Histogram and ANOVA of log grooming rates}

Although the primary goal of this study is in assay development, we examined the grooming behavior of three commercial stocks of bees to calculate their grooming rates as a proof-of-concept. We chose to study Russian, Italian, and Minnesota Hygienic honey bees, since the Russian and Minnesota Hygienic bees are bred for their superior hygienic traits (Spivak and Reuter, 2001). We wanted to explore if these two stocks have statistically significant higher grooming rates than the Italian bees, which are known for their relatively high honey production and low defensiveness. The hygienic behaviors of the Russian and Minnesota Hygienic bees have previously been shown to be quantitatively greater than that for the Italian bees (Spivak and Reuter, 2001). If the grooming rates of the Russian and Minnesota Hygienic bees are equivalent to the rate obtained from the Italian bees, then this would suggest that hygienic behavior and grooming behavior are uncoupled at the individual and colony levels.

In order to explore the null hypothesis that different honey bee commercial stocks exhibit different grooming rates, here, denoted $k$, analysis of the time until clean $(1 / k)$ and a means comparison test of log grooming rates was performed. Figure 4a shows that approximately $94 \%$ of the 96 grooming experiments were complete in seven minutes or less. This percent changes to approximately $88 \%$ in five minutes or less. The null hypothesis that different stocks exhibit different grooming rates was tested with a one-way ANOVA, and was rejected with a p-value of $p=0.13$ (Table 1). A swarm plot of the respective stock log grooming rates (Figure $4 \mathrm{~b}$ ) gives visual confirmation of the overall equivalence of grooming rates between different stocks (plot routine from the MATLAB File Exchange program plotSpread; Jonas, 2012). However, it is possible that there are subtle differences, such as a markedly narrower distribution for the Russian stock. Although the mean $\varphi_{0}$ was different for the three stocks $(p=.3)$, since the starting flour coverage was parameterized by experiment, that experiment should uniquely define the resulting grooming rates. In this pilot study, there are no statistically significant 
differences in the grooming rates between stocks. Larger studies may find differences not evident in this present study.
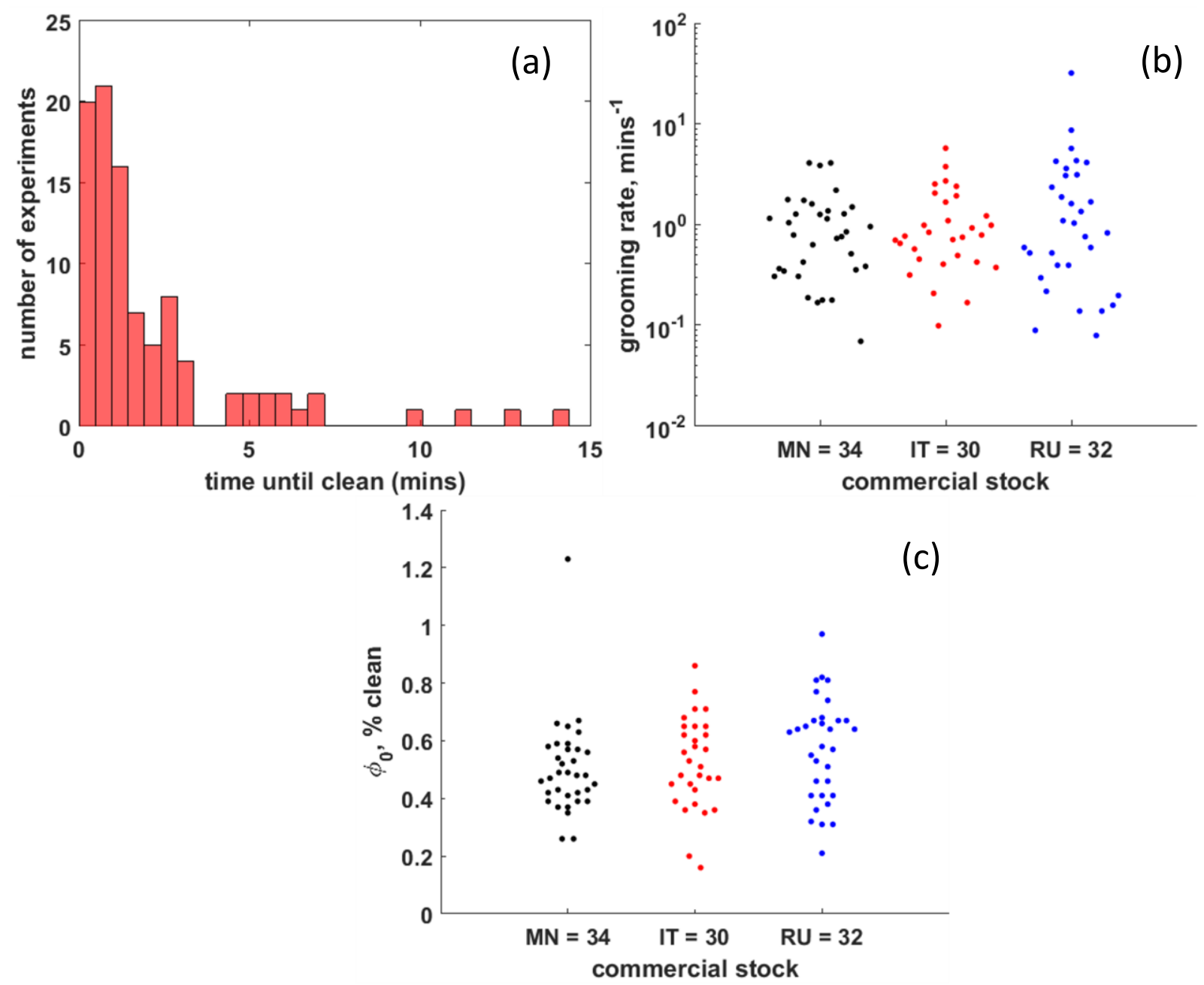

Figure 4 (a) Histogram of time until clean and (b) swarm plot of log grooming rate versus commercial stock. Differences between stocks are not statistically significant, but there are minor variations in the distributions of grooming rates. (c) Mean initial cleanliness ratio phi0 differs between stocks $(p=.3)$. 
Table 1. ANOVA of log grooming rates.

\begin{tabular}{|l|l|l|l|l|l|}
\hline Source & SS & df & MS & F & Prob>F \\
\hline Groups & 51 & 2 & 26 & 2.1 & 0.13 \\
\hline Error & 1139 & 93 & 12 & & \\
\hline Total & 1190 & 95 & & & \\
\hline
\end{tabular}

\section{Discussion}

Dead or diseased honey bee brood are a critical factor in determining the overall health of a honey bee colony. The Minnesota Hygienic bees are a special commercial stock of honey bees, selectively bred for their ability to remove dead honey bee brood from the hive (Spivak and Reuter, 2001). This behavior leads to a healthier colony, as harbors of diseases and pathogens are quickly removed from the hive before they have the opportunity to spread.

Grooming is another important aspect of honey bee health. Honey bees that exhibit high levels of grooming should be less susceptible to diseases than bees that favor other behaviors (Arechavaleta-Velasco et al., 2012). One goal of this study was to test whether hygienic honey bees are any better at grooming than bees whose hygienic behaviors are unknown. Since this hypothesis was rejected, it implies that more work needs to be done on the study of honey bee grooming as a function of social immunity, independently from hygienic behavior. Our results demonstrate, however, that the underlying genetic mechanisms that govern hygienic behavior (Lapaidge et al., 2002) are likely distinct and uncoupled from those underlying grooming behavior.

This assay is an important step towards studying honey bee behaviors that have been largely neglected due to the amount of labor needed to acquire even a basic understanding of bee ethology. Coating bees in baking flour is an extraordinarily cheap, quick, and safe way to instigate autogrooming behavior. Most scientists have access to recording equipment and 
personal computers, so this assay can be easily replicated in any lab. The most challenging aspect of this setup is the lighting; special care needs to be taken to ensure the recording area has proper illumination, without causing reflective glare from the petri dish or shadows cast by the honey bees.

There are multiple changes that can be made to our experimental protocol to ensure greater success in the future. Although much care was taken to ensure that bees were coated in flour and transferred to the recording arena immediately, it appeared that much of the grooming occurs within the first two to four minutes of the recording, and as such parameterization of initial bee cleanliness was necessary. The two-minute acclimation buffer may be unnecessary, and certainly could be shortened, but direct experimentation would be required. Moreover, across all of our experiments, it seems clear that 12 minutes of recording time was far more than necessary. All of our experimental timeframes could be shortened conservatively to $\sim 6$ minutes, making this method even more efficient than originally anticipated.

One possible explanation for the different mean $\varphi_{0}$ across stocks might be effects of temperature or humidity on the days each experiment was performed, but neither factor predicted parameter fits $(p>0.3)$. A different explanation might be differences in hair coverage between the stocks. The honey bee exoskeleton is covered with thousands of macro- and microscopic setae (or simply, hairs) that both provide sensory information from the environment function and facilitate pollen foraging (Winston, 1987). Bee hair was not measured, but it is plausible that there are consistent differences in hair between stocks.

Honey bees are eusocial insects, and prior to running the experiments, it seemed intuitive to film experiments as groups of five honey bees, in case both allo- and autogrooming events were observed. Unfortunately, it turned out that the observed behavior was quite different than originally hypothesized. Honey bees that were overwhelmed with external stimuli, in fact, exhibited extremely selfish behavior, and seemed to focus on autogrooming entirely. To 
this end, it would be beneficial to build on this experimental design by focusing on one bee at a time, rather than on groups of five. Since the first six minutes of this protocol are critical for examining honey bee grooming behavior, this change in protocol would facilitate a faster turnover between the time the bee has been coated in flour and the time the recording process begins. That said, pooling groups of five was intentionally done as a means of controlling for variability of individuals belonging to a significantly larger collective. Comparisons between individual versus pooled protocols need to be made. A modified experimental design that might capture both allo- and autogrooming behaviors might be adding a single bee coated in flour to a dish with one (or more) uncoated bees. This design might allow both grooming behaviors to be studied at the same time, more accurately capturing the overall grooming rate in social insects.

The chosen model has reasonable assumptions, and characterizes the typical forms of these time series. Although other models (not included in this study), were tested, they were rejected either due to their complexity, or because they were lacking a parameter describing how clean the bees were at the start of recording $\left(a_{0}\right)$. One small issue with the selected mathematical model is that the data may start above the asymptotic value of a clean honey bee because of stochastic noise. For example, one curve started at a value of $120 \%$ (Fig. 3d). Since our model is based on a differential equation, the fit cannot pass below the asymptotic value of $100 \%$. This type of curve can occur when bees clump together at the start of the experiment, resulting in the artificial illusion that the bees are becoming dirtier. This event only occurred once, however, and was treated as an outlier.

\section{Conclusion}

Image thresholding could be applied in any system whereby an organism can be recorded against a sufficiently contrasting solid background. Grooming can be measured as a function of time. While more research is required to directly measure honey bee grooming, it is clearly an important yet understudied aspect of social immunity. Many current assays only 
measure grooming as a function of parasite removal. Hopefully, the novel assay described here will lead to additional empirical studies on this topic, or successful breeding programs. Future work could be expanded to other insects such as ants, wasps, and other bee species.

\section{Conflict of interest}

No conflict of interest.

\section{Acknowledgements}

We would like to thank Dr. Michael Simone-Finstrom and Dr. Uduak George for their support; Jennifer Keller for her dedicated work at the Lake Wheeler honey bee facility; and undergraduates Paulina Spencer, Jamal Moss, and Jason Brannock for their work on this project. This project was supported by the UBM-Group: Integrated Undergraduate Training in Mathematics and Life Sciences at NCSU (DBI-1129214) and a grant from USDA-APHIS (148130-0360-CA) awarded to DRT.

\section{References}

1. Andino, Gladys K., and Greg J. Hunt. "A scientific note on a new assay to measure honeybee mite-grooming behavior." Apidologie 42.4 (2011): 481-484.

2. Arechavaleta-Velasco, Miguel E., et al. "Fine-scale linkage mapping reveals a small set of candidate genes influencing honey bee grooming behavior in response to Varroa mites." PLoS One 7.11 (2012): e47269.

3. Arechavaleta-Velasco, Miguel, and Ernesto Guzmán-Novoa. "Relative effect of four characteristics that restrain the population growth of the mite Varroa destructor in honey bee (Apis mellifera) colonies." Apidologie 32.2 (2001): 157174. 
4. Aumeier, Pia. "Bioassay for grooming effectiveness towards Varroa destructor mites in Africanized and Carniolan honey bees." Apidologie 32.1 (2001): 81-90.

5. Büchler, Ralph, et al. "Standard methods for rearing and selection of Apis mellifera queens." Journal of Apicultural Research 52.1 (2013): 1-30.

6. Cremer, Sylvia, Sophie AO Armitage, and Paul Schmid-Hempel. "Social immunity." Current biology 17.16 (2007): R693-R702.

7. Evans, Jay D., and Marla Spivak. "Socialized medicine: individual and communal disease barriers in honey bees." Journal of Invertebrate Pathology 103 (2010): S62-S72.

8. Guzman-Novoa, Ernesto, et al. "Genotypic variability and relationships between mite infestation levels, mite damage, grooming intensity, and removal of Varroa destructor mites in selected strains of worker honey bees (Apis mellifera L.)." Journal of invertebrate pathology 110.3 (2012): 314-320.

9. Hardeberg, Jon. Acquisition and reproduction of color images: colorimetric and multispectral approaches. Universal-Publishers, 2001.

10. Jonas. Plot spread points (beeswarm plot). MATLAB Central File Exchange. Retrieved June 1, 2016.

11. Lapidge, K. L., B. P. Oldroyd, and M. Spivak. (2002). Seven suggestive quantitative trait loci influence hygienic behavior of honey bees. Naturwissenschaften, 89: 565-568.

12. Pritchard, Dorian J. "Grooming by honey bees as a component of varroa resistant behavior." Journal of Apicultural Research (2016): 1-11.

13. Schmid-Hempel, P. (1998). Parasites in Social Insects. Princeton, N. J.: Princeton University Press, pp 409.

14. Schneider, Caroline A., Wayne S. Rasband, and Kevin W. Eliceiri. "NIH Image to ImageJ: 25 years of image analysis." Nat methods 9.7 (2012): 671-675. 
15. Simone, Michael, Jay D. Evans, and Marla Spivak. "Resin collection and social immunity in honey bees." Evolution 63.11 (2009): 3016-3022.

16. Spivak, Marla, and Gary Reuter. "Resistance to American foulbrood disease by honey bee colonies Apis mellifera bred for hygienic behavior." Apidologie 32.6 (2001): 555-565.

17. Tu, Gang Jun, et al. "Automatic behaviour analysis system for honeybees using computer vision." Computers and Electronics in Agriculture 122 (2016): 10-18.

18. Winston, Mark L. The biology of the honey bee. Harvard University Press, 1987. 